\title{
El método científico y la nueva filosofía de la ciencia: aportes y perspectivas
}

José Eriberto Cifuentes Medina *

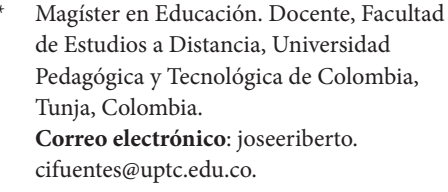

Recibido: 3 de junio del 2016

Aprobado: 24 de agosto del 2016

Cómo citar este artículo: Cifuentes, José. "El método científico y la nueva filosofía de la ciencia: aportes y perspectivas". Rastros Rostros 18.33 (2016): xx-xx. Impreso. doi: http://dx.doi.org/10.16925/ra.v18i33.1681

\section{Resumen}

Propósito: el quehacer de la filosofía de la ciencia y el proceso arquitectónico de los paradigmas tiene como objetivo primordial la construcción racional de un conjunto de paradigmas correspondientes a las inquietudes propias de la filosofía. Descripción: lo concerniente al tratado de la filosofía de la ciencia, permite inferir que la filosofía y la ciencia se ocupan del saber y de cómo se desarrollan, evalúan y cambian las teorías científicas, y de saber, y si la ciencia es capaz de revelar la verdad de las entidades ocultas y los procesos de la naturaleza, es decir, si es el proceso de concordancia de la intelección con la realidad permitiendo procesos de aprendizaje y reflexión como su objeto de estudio. Punto de vista: entre filosofía y ciencia se da un proceso de conocimiento en el que intervienen tres elementos: un sujeto, un objeto y una relación que se establece entre los dos. Conclusiones: se deja en firme la importancia de la filosofía de la ciencia en las investigaciones de diversos campos en la actualidad. Se logra enaltecer la relación entre la filosofía y la ciencia, teniendo en cuenta la posición de algunos filósofos y científicos que lo han logrado para efectos de la formación filosófica y científica de los seres humanos.

Palabras clave: ciencia, filosofía, método científico. 


\title{
The scientific method and the new philosophy of science: contributions and perspectives
}

\begin{abstract}
Purpose: The main objective of the philosophy of science and the architectural process of paradigms is the rational construction of a set of paradigms that correspond to the concerns of philosophy. Description: That which concerns the philosophy of science treatise, allows to infer that philosophy and science deal with knowledge; with how scientific and knowledge theories are developed, evaluated and changed; with whether science is capable of revealing the truth of hidden entities and the processes of nature, that is, if there is concordance between intellection and reality that allows processes of learning and reflection. Point of view: between philosophy and science there is a process of knowledge in which three elements are involved: a subject, an object and the relationship established between the two. Conclusions: the importance of the philosophy of science in the researches of diverse fields in the present time is confirmed. The relationship between philosophy and science is enhanced, taking into account the case of some philosophers and scientists who have achieved this for the philosophical and scientific formation of human beings.
\end{abstract}

Keywords: science, philosophy, scientific method.

\section{O método científico e a nova filosofia da ciência: contribuições e perspectivas}

\section{Resumo}

Propósito: o fazer da filosofia da ciência e o processo arquitetônico dos paradigmas têm como objetivo primordial a construção racional de um conjunto de paradigmas correspondentes às inquietudes próprias da filosofia. Descrição: o concernente ao tratado da filosofia da ciência permite inferir que a filosofia e a ciência se ocupam do saber e de como se desenvolvem, avaliam e mudam as teorias científicas e do saber, e se a ciência é capaz de revelar a verdade das entidades ocultas e os processos da natureza, ou seja, se é o processo de concordância da intelecção com a realidade permitindo processos de aprendizagem e reflexão como seu objeto de estudo. Ponto de vista: entre filosofia e ciência se dá um processo de conhecimento no qual intervêm três elementos: um sujeito, um objeto e uma relação que se estabelece entre os dois. Conclusões: ressalta-se a importância da filosofia da ciência nas investigações de diversos campos na atualidade. Consegue-se enaltecer a relação entre a filosofia e a ciência, levando em consideração a posição de alguns filósofos e pesquisadores que o fizeram para efeitos da formação filosófica e científica dos seres humanos.

Palavras-chave: ciência, filosofia, método científico. 


\section{Introducción}

La epistemología es la disciplina que puede mediar entre la filosofía y ciencia. Esta se encarga de determinar los tipos de interacción que se dan entre los dos elementos anteriormente mencionados, cuando de su relación surge un tipo de conocimiento que se llama científico, se configura el análisis del conocimiento que surge de que la filosofía ejercerá su función en relación con la ciencia y la pregunta, sobre el porqué de sus inquietudes y cómo hacer para llegar al conocimiento. El método científico y la nueva filosofía de la ciencia, son la hermenéutica que ahora compete disertar para la comprensión y su aplicación a la vida misma y la academia, como posibilidad de acceder al conocimiento y de resolver los interrogantes que se plantea el ser humano en la realidad.

Los postulados de Azcárraga (2), entre otros académicos, permiten aducir la relación entre filosofía y ciencia, en la connotación del sustrato filosófico de las teorías científicas, por lo que no se podría afirmar que la una se pueda constituir en superior y la otra en inferior. Dado que la relación de sujeto, objeto y la relación entre los dos es propia en la filosofía y también de la ciencia por los postulados propios de su correlación; por ejemplo, una teoría científica en primer momento pudo ser una inspiración filosófica y existe una clara influencia de los filósofos en los científicos como Descartes a Newton, entre otros.

Los planteamientos de Asensi-Artiga y ParraPujante (4) llevan a fijar los nuevos planteamientos de la filosofía de la ciencia y el desarrollo del método científico. Los esbozos que se realizan se acercan a la dirección de una nueva filosofía de la ciencia, en aras de plantear nuevas ideas, pues la filosofía no es estática, y más aún, la filosofía de la ciencia es dinámica, según se evidencia en los diversos postulados.

\section{Importancia de la ciencia y la filosofía como fuente y generación de conocimiento}

El artículo hace un acercamiento al engranaje entre filosofía y ciencia, como posibilidad de fuente y generación de conocimiento a lo largo de la historia y su acentuada connotación desde el siglo XVI. La relación entre el filósofo y el científico, se puede inferir, es cercana dadas las circunstancias de implicación epistemológica y ontología que las entrelaza. Para un primer acercamiento entre filosofía y ciencia, se señala la apreciación de la definición de la Real Académica Española (tabla1).

Tabla 1. Definición de la Real Académica Española

\begin{tabular}{|c|c|}
\hline CIENCIA & FILOSOFÍA \\
\hline $\begin{array}{l}\text { 1. Conjunto estructurado de conocimientos obtenidos mediante la } \\
\text { observación y el razonamiento, de los que se derivan principios y leyes } \\
\text { generales. La ciencia ha experimentado un avance enorme. 2. Cada una de } \\
\text { las ciencias que tiene un método o un objeto de estudio determinados. } \\
\text { Ciencias humanas y sociales: cuyo objeto de estudio es alguna actividad } \\
\text { intelectual o social del ser humano, como la psicología, la filosofía o la } \\
\text { antropología } \\
\text { Ciencias naturales: cuyo objeto de estudio es algún aspecto de la naturaleza } \\
\text { y sus fenómenos, como la geología, la botánica o la zoología } \\
\text { Ciencias ocultas: conjunto de conocimientos y practicas secretas, como la } \\
\text { magia, la alquimia o la astrología, que pretenden desvelar misterios de la } \\
\text { naturaleza y que no están consideradas como ciencias }\end{array}$ & $\begin{array}{l}\text { 1. Ciencia que busca establecer, de manera racional, los } \\
\text { principios más generales que organizan y el orientan el } \\
\text { conocimiento de la realidad, así como el sentido del obrar } \\
\text { humano } 2 \text {. Conjunto sistemático de concepciones de } \\
\text { filosofía - de alguien - La filosofía de Kant. 3. Manera } \\
\text { de pensar de alguien, o concepción que tiene de la vida y } \\
\text { de las cosas. Mi filosofía se resume en el lema: vive y deja } \\
\text { vivir. }\end{array}$ \\
\hline
\end{tabular}

Fuente: Real Academia Española.

También presento una breve descripción o esbozo general de las diversas ciencias a fin de ubicar la filosofía, esta se encuentra entre las ciencias humanas según Contreras (3), y según la Real Academia Española (tabla 2), de igual forma por lo que coincide que la Filosofía es una ciencia y que se ubica entre las ciencias sociales y humanas. En la tabla 2 se refleja el orden que propone Contreras.
Se puede constatar una vez más que la filosofía es una ciencia pero que se puede hacer filosofía de la ciencia y por ello el filosofar es pensar y/o reflexionar filosóficamente sobre algo, que para el caso es: la ciencia. Las dos están relacionadas con el conocimiento, como eje central de análisis. 
Tabla 2. Saber humano dividido en cuatro grandes grupos

\begin{tabular}{|l|l|}
\hline \multicolumn{1}{|c|}{ CIENCIA } & \multicolumn{1}{|c|}{ DEFINICIÓN } \\
\hline Ciencias Formales & $\begin{array}{l}\text { De manera general corresponde a las matemáticas y la lógica. Estas no estudian objetos empíri- } \\
\text { cos sino lenguajes de signos en donde lo que cuenta es la sintaxis de esos lenguajes, es decir, la } \\
\text { manera como se relacionan entre sí. }\end{array}$ \\
\hline Ciencias Naturales & $\begin{array}{l}\text { Estas estudian todas las cosas y procesos que existen con independencia del ser humano y que } \\
\text { son conocidas mediante métodos matemáticos, experimentales y observaciones }\end{array}$ \\
\hline Ciencias Sociales & $\begin{array}{l}\text { Estas estudian al ser humano en sociedad y la legalidad que es propia de las relaciones sociales. } \\
\text { A este grupo pertenecen: la economía, la administración y la sociología, como las disciplinas } \\
\text { más destacadas. }\end{array}$ \\
\hline Ciencias Humanas y Humanidades & $\begin{array}{l}\text { En las Ciencias Humanas encontramos la Antropología, la lingüística, la etnología, etc. Las } \\
\text { humanidades comprenden la historia, las artes, la literatura, la filosofía, la teoría de la religión, } \\
\text { la música, etc. A ello hay que agregar los saberes nuevos como el séptimo arte, o sea el cine, las } \\
\text { ciencias de la comunicación, la semiótica, etc. Con esto explicamos la parte de la definición } \\
\text { que afirma que las humanidades son una subdivisión del saber humano. }\end{array}$ \\
\hline
\end{tabular}

Fuente: información recabada de Contreras.

La filosofía se consolida como fuente y generación de conocimiento, se considera que se da bajo observación y se requiere de una estructura racional. Surge, entonces, la siguiente pregunta ¿cuál es el papel de la filosofía en los inicios del siglo xxi y del tercer milenio? y Bojacá responde: se le asigna a la filosofía el papel de construir sinopsis de sistemas de conocimientos producidos por la ciencia, así la filosofía es una metaciencia. La metafilosofía es el análisis de la actividad filosófica por medio del lenguaje filosófico (Bojacá 168).

A lo largo de la historia se han dado muchos giros al concepto de filosofía, sin embargo, se remarca la concepción de los primeros filósofos griegos, quienes optaron por llamarse amigos de la sabiduría, estudiosos y aficionados a la conciencia socrática: "solo sé que nada sé". Veamos algunos de los primeros filósofos y su planteamiento resumido en la tabla 3.

Tabla 3. Antecedentes de la filosofía

\begin{tabular}{|l|l|}
\hline \multicolumn{1}{|c|}{ FILÓSOFO } & \multicolumn{1}{c|}{ PROPUESTA } \\
\hline Pitágoras de Samos (582-500 a.C.) & La filosofía es un afán de saber libre y desinteresado. \\
\hline Sócrates (469-399 a. C.) & $\begin{array}{l}\text { La filosofía es un afán que siente el hombre por saber de sí mismo (conócete a ti mismo). } \\
\text { La filosofía es el amor permanente a la sabiduría y la búsqueda de la verdad de las cosas; pues la } \\
\text { sabiduría misma es patrimonio de los dioses. } \\
\text { Filosofía es la búsqueda de la verdad como medida de lo que el hombre debe hacer y como } \\
\text { norma para su conducta. }\end{array}$ \\
\hline Platón (427-347 a. C.) & $\begin{array}{l}\text { La filosofía es la ciencia de la razón de las cosas. La filosofía es la más alta ascensión de la perso- } \\
\text { nalidad y la sociedad humana por medio de la sabiduría. }\end{array}$ \\
\hline Aristóteles (384-322 a. C.) & $\begin{array}{l}\text { Todo lo que sobre este punto nos proponemos decir ahora, es que la ciencia que se llama filoso- } \\
\text { fía es, según la idea que generalmente se tiene de ella, el estudio de las primeras causas y de los } \\
\text { principios. La filosofía es la ciencia del ser en tanto que ser. } \\
\text { Es imprescindible que sea la ciencia teórica de los primeros principios y de las primeras causas, } \\
\text { porque una de las causas es el bien, la razón final. Y que no es una ciencia práctica, lo prueba el } \\
\text { ejemplo de los primeros que han filosofado. Lo que en un principio movió a los hombres a hacer } \\
\text { las primeras indagaciones filosóficas fue como lo es hoy la admiración. }\end{array}$ \\
\hline
\end{tabular}

Fuente: información recabada de Valdés.

La filosofía es una actividad mental por medio de la cual construimos el laberinto armónico de las ideas, muchas de ellas cargadas de vivencias y algunas tendientes a la práctica. Para Wittgenstein, la filosofía no es una ciencia sino una actividad. La palabra filosofía connota un sentido dinámico, de desconstrucción, de repensamiento, de construcción (Bojacá
183). La pregunta es la puerta que nos introduce a la reflexión y a la posterior repuesta. Lo problémico suscita la inquietud problematizadora a nivel mental y la consiguiente reflexión bajo la vivencia de la verdad. De la pregunta nacen la investigación científica y la reflexión filosófica. 
Si de la pregunta nace la investigación y la reflexión, ahora la cuestión es: ¿por qué surge la filosofía de la ciencia? La respuesta en la voz de Valdés:

- Entre los objetos que admiraban y no podían darse razón, los hombres, se aplicaron primero a los que estaban a su alcance; después, quisieron explicar los más grandes fenómenos; por ejemplo, las diversas fases de la luna, el curso del sol y de los astros, y por último, la formación del universo

- La filosofía de la ciencia investiga el conocimiento científico y la práctica científica.

- Son filosóficas las diversas proposiciones básicas que permiten construir la ciencia

- La filosofía de la ciencia intenta explicar problemas tales como: la naturaleza y la obtención de las ideas científicas (conceptos, hipótesis, modelos, teorías, paradigmas, etc.) Y la relación de cada una de ellas con la realidad. (189)

En relación con la discusión de la cuestión en mención se deriva la pregunta ¿de qué manera ha influido el problema del conocimiento en la historia y qué importancia tiene dedicarnos a su análisis? A la filosofía no le conviene perder el terreno que ha logrado ganar en la historia frente a la ciencia, sin embargo, no ha se ser la esclava, pero tampoco la ama, ha de estar en correlación y ocupando el lugar que le corresponde de frente a la ciencia.

Por lo tanto, surgen preguntas ¿qué es conocimiento?, ¿en qué se funda el conocimiento?, ¿es posible conocer? Estas cuestiones han ocupado el trabajo de muchos filósofos, entre tanto, numerosos científicos, se han dado por satisfechos dejando la filosofía de la ciencia a los filósofos y han preferido seguir haciendo ciencia en vez de dedicar más tiempo a considerar cómo se hace la ciencia. Cada uno desde su perspectiva y desde sus posibilidades de explicación ha intentado resolverlas. Cada respuesta abre una nueva discusión, por lo que este campo filosófico es bastante amplio.

En el momento de abordar el problema sobre la forma como se debe llamar a la disciplina que trata este tipo de reflexiones filosóficas, no se ha llegado a un consenso, se le podría denominar: gnoseología, epistemología, teoría del conocimiento o crítica del conocimiento, como tratados de la filosofía y que ahora confluyen en filosofía en la filosofía de la ciencia y apoya a la filosofía ahora en dirección de filosofía de la ciencia. La definición más común de conocimiento plantea que es la aprehensión intelectual de un objeto por parte de un sujeto. Sin embargo, esta misma definición ha tenido muchos detractores a través de la historia y son muchos los que han buscado darle un sentido diferente para que dicho proceso no parezca tan mecánico o tan intelectivo (Verano 182). Otros se han dedicado a determinar cómo es que se da tal proceso y hasta donde es realmente posible que se dé, también hay quienes han pretendido encontrar el tipo de sujeto que conoce y el tipo de conocimiento que se puede obtener en diferentes contextos y en relaciones concretas entre el sujeto y el objeto.

Las preguntas que las personas se formulan de manera frecuente referentes al problema del conocimiento son ¿es posible conocer?, ¿cuál es el origen del conocimiento?, ¿cuál es la esencial del conocimiento?, ¿cuántas y cuales formas de conocimiento hay?, ¿cuáles son los criterios de validez y de verdad del conocimiento?, ¿qué hace que a un conocimiento se le denomine conocimiento científico? (Suárez 12). Las respuestas que se han dado a través de la historia han sido variadas, desde la antigüedad griega se han intentado resolver en la perspectiva de Sócrates, Platón, Aristóteles, al igual que en la Edad Media, Moderna y hasta en la actualidad, aún continua la discusión de la cuestión con los planteamientos propios, según los argumentos desde la posición de cada quien y el paradigma sobre el cual se encuentre. En la perspectiva de Azcárraga (5), se expone que:

Fue el filósofo, matemático y maestro de Bertrand Russell, Alfred N. Whitehead, quien afirmó que toda la historia de la filosofía podría reducirse a notas de pie de página a los diálogos platónicos. La frase, aunque exagerada, cobra su verdadero sentido si recordamos que Platón vivió hace 2400 años. La filosofía no puede, hoy, vivir de espaldas al conocimiento científico, y menos aún, tratar de relativizarlo en un intento fútil de retener un espacio privilegiado o protegido, que es lo que, en el fondo, han intentado algunos filósofos postmodernos con sus críticas desmesuradas a la racionalidad y objetividad de la ciencia. La filosofía debe estar, hoy, unida a la ciencia. La ciencia nos ayuda a comprender cómo son las cosas, no cómo nos parecen o desearíamos que fueran, y por tanto es, a largo plazo, más inmune a nuestras preconcepciones, filias y fobias.

En relación con la ciencia, Popper, defensor de su racionalidad, consideraba que esta es probablemente la única actividad humana en la que los errores son 
criticados sistemáticamente $\mathrm{y}$, finalmente, corregidos (Azcárraga 6). Autor que en un primer momento expone el origen de la filosofía haciendo una fuerte pero necesaria crítica, pasando luego al campo de la discusión que nos interesa, se trata del debate que al respecto surge entre Popper y Wittgenstein, y luego a la puesta en marcha del sustrato filosófico de las teorías científicas, a lo largo de la historia.

Por lo tanto, se ha de observar que la filosofía de la ciencia se puede describir cómo aquella que lleva a cabo la investigación y la forma en que debe hacerse y que se estudia ¿cómo se relacionan entre sí el mundo real, la información empírica, los modelos y las teorías y qué se puede hacer para mejorar su relación? Se suele considerar que la filosofía de la ciencia alcanza su edad adulta en los años de 1920 con la aparición del Círculo de Viena, integrado por un grupo de filósofos: Rudolf Carnap (1891-1970), Otto Neurath (1881-1945), Hans Hahn (1879-1934), Kurt Gödel (1906-1978), Willard V. Quine (1908-2000). La filosofía se la ciencia transciende como un hecho histórico del siglo xx y ahora se proyecta en el siglo xxI, dejando retos de inmensurable trabajo para los filósofos y para los científicos.

\section{El método científico y la nueva filosofía de la ciencia}

La disertación, con el apoyo de Asensi-Artiga y ParraPujante (8), Valdés (2002), Bojacá (182), Suárez (14), entre otros, se organiza en dos apartados: el primero pretende hacer un bosquejo rápido y a manera de línea del tiempo de la filosofía de la ciencia desde el siglo xv hasta el xx y el segundo se dedica al método científico como constitutivo junto a la investigación científica de la nueva filosofía de la ciencia.

\section{Hacia una nueva filosofía de la ciencia}

En aras de avanzar en la disertación de la filosofía de la ciencia y la nueva filosofía de la ciencia en apoyo del método científico, se pretende, en primer lugar, hacer un trazado general y sistemático de la historia de la ciencia, con el propósito de acceder a los postulados de Asensi-Artiga y Parra-Pujante (9), quienes afirman que su artículo:

no pretende ser un esbozo de filosofía de la ciencia, sino algo más modesto: un rápido repaso a los caminos de esta a lo largo del siglo XX recién acabado con el fin de mostrar, tras el giro lingüístico que la filosofía dio a comienzos de esa centuria con autores como Rusell o el llamado 'primer Wittgenstein', el autor del Tractatus Logico- Philosophicus, ciertos jalones de la nueva filosofía de la ciencia, hasta llegar a la orientación que podríamos llamar historicista, para cuyos defensores más extremos, como Feyerabend, habría que proceder a una especie de desconstrucción que despejara las verdaderas razones de uno u otro paradigma.

Antes de abordar la ocupación de Asensi-Artiga y Parra-Pujante (9), se acude de manera general y resumida a la historia de la filosofía (tabla 4) como antecedente de la filosofía de la ciencia. La primera tiene sus raíces en la filosofía griega y surgió como una disciplina autónoma en el siglo xIx. Aunque ya a partir de antes, filósofos e investigadores se interesaron en la filosofía de la ciencia, desde épocas remotas.

Tabla 4. Breve historia de la filosofía de la ciencia (siglos XV, XVI, XVII, XIX y Xx)

\begin{tabular}{|c|c|}
\hline AUTOR & PLANTEAMIENTO \\
\hline Nicolás Copérnico (1473 - 1543,Polonia) & Fue un astrónomo del renacimiento que formuló la teoría heliocéntrica del sistema solar \\
\hline Galileo Galilei (1564-1642, Italia) & $\begin{array}{l}\text { Sus logros incluyen la mejora del telescopio, gran variedad de observaciones astronómicas, } \\
\text { la primera ley del movimiento y un apoyo determinante a la teoría de Copérnico. }\end{array}$ \\
\hline Francis Bacon (1561-1626, Inglaterra) & $\begin{array}{l}\text { Es considerado uno de los padres del empirismo, sus obras y pensamientos ejercieron una } \\
\text { influencia decisiva en el desarrollo del método científico. }\end{array}$ \\
\hline Johannes Kepler (1571-1630, Alemania) & $\begin{array}{l}\text { Conocido fundamentalmente por sus leyes sobre el movimiento de los planetas en su órbita } \\
\text { alrededor del sol. }\end{array}$ \\
\hline Isaac Newton (1643-1727, Inglaterra) & $\begin{array}{l}\text { Planteó la ley de la gravitación universal y estableció las bases de la mecánica clásica } \\
\text { mediante las leyes que llevan su nombre, también se destacan sus trabajos sobre la natura- } \\
\text { leza de la luz y la óptica y el desarrollo del cálculo matemático. }\end{array}$ \\
\hline Albert Einstein (1879-1955, Alemania) & $\begin{array}{l}\text { Presentó la teoría de la relatividad general, en la que reformuló por completo el concepto de } \\
\text { gravedad. Estudio científico del origen y la evolución del universo por la rama de la física } \\
\text { denominada cosmología. }\end{array}$ \\
\hline
\end{tabular}

Fuente: información recabada y reorganizada de Valdés (30). 
En el siglo XviII, la filosofía de la ciencia era considerada como una disciplina autónoma y podía ser vista como una manera de describir cómo se lleva a cabo la investigación, con Augusto Comte cambian los paradigmas de la filosofía y de la ciencia y también otros filósofos y científicos realizan sus planteamientos, podemos observar un resumen en la tabla 5.

Tabla 5. Breve historia de la filosofía de la ciencia (siglo XVIII)

\begin{tabular}{|l|l|}
\hline \multicolumn{1}{|c|}{ AUTOR } & \multicolumn{1}{c|}{ PLANTEAMIENTO } \\
\hline $\begin{array}{l}\text { Augusto Comte (1798-1857, } \\
\text { Francia) }\end{array}$ & $\begin{array}{l}\text { Creó la palabra altruismo (dar sin esperar nada a cambio) su filosofía tuvo gran repercusión que se } \\
\text { fundaron países como Brasil en función de su filosofía, país en cuya bandera se lee "Orden y Pro- } \\
\text { greso", parte de la triada filosófica de Comte (altruismo, orden, progreso). Miraba a la ciencia como } \\
\text { una fuente de progreso, la forma en la que organizamos las universidades, etc. }\end{array}$ \\
\hline $\begin{array}{l}\text { John Stuart Mill (1806-1873, } \\
\text { Inglaterra) }\end{array}$ & $\begin{array}{l}\text { Fue un seguidor del positivismo de Comte, también fue representante del pensamiento romántico y } \\
\text { del socialismo. }\end{array}$ \\
\hline $\begin{array}{l}\text { David Hume (1711-1776, } \\
\text { Inglaterra) }\end{array}$ & $\begin{array}{l}\text { Planteó que el conocimiento científico es meramente probable, no necesario. } \\
\text { Numerosos científicos, sin embargo, se han dado por satisfechos dejando la filosofía de la ciencia a los } \\
\text { filósofos y han preferido seguir haciendo ciencia en vez de dedicar más tiempo a considerar cómo se } \\
\text { hace la ciencia. El debate realismo-anti realismo es un debate filosófico que explora los fundamentos } \\
\text { de la verdad científica comúnmente aceptada. } \\
\text { Por su parte, el reduccionismo científico es una idea muy debatida en la filosofía de la ciencia, en el } \\
\text { que la ciencia reduce las interacciones y entidades complejas a la suma de las partes que la componen. }\end{array}$ \\
\hline
\end{tabular}

Fuente: información recabada y reorganizada de Valdés (35).

Numerosos científicos, sin embargo, se han dado por satisfechos dejando la filosofía de la ciencia a los filósofos y han preferido seguir haciendo ciencia en vez de dedicar tiempo a considerar cómo se hace la ciencia. Según Valdés (32) y los postulados de AsensiArtiga y Parra-Pujante (10), la filosofía de la ciencia alcanza su edad adulta en los años veinte con la aparición del Círculo de Viena. El Círculo de Viena, encabezado por Johan Craidoff (1899-1960, Alemania), propuso un modelo de ciencia en el que esta procede mediante generalizaciones (inducción) a partir de los datos. Propuso la idea central del positivismo y del neopositivismo en el cual la ciencia debe utilizar las teorías como instrumentos, para predecir fenómenos observables y debe renunciar a buscar explicaciones. La búsqueda de explicaciones es función de la metafísica, que no es ciencia sino palabrería carente de significado.

Tabla 6. Definición de ciencia según varios autores
Con el progreso de la ciencia, esta comenzó el estudio de campos que están más allá de la experiencia, como puede ser la física de altas energías o la física atómica. En esta situación, el criterio empirista de verdad condujo a muchos problemas, lo que llevó a diversas matizaciones del mismo. El verificacionismo estricto acabó siendo abandonado y sustituido por la contrastación entre proposiciones y observaciones, lo que permite una confirmación gradualmente creciente de las teorías.

Asensi-Artiga y Parra-Pujante (10) dicen que es necesario iniciar los estudiantes en el conocimiento de la investigación científica, introducirlos en el estudio de la naturaleza de la ciencia y de todos aquellos elementos que permiten el desarrollo de sus objetivos, evocando a autores como Sierra, Bunge y Kohan, un breve resumen en la tabla 6 .

\begin{tabular}{|l|l|}
\hline \multicolumn{1}{|c|}{ AUTOR } & \multicolumn{1}{c|}{ DEFINICIÓN } \\
\hline Sierra Bravo & $\begin{array}{l}\text { Define la ciencia en sentido estricto, como un conjunto sistemático de conocimientos sobre la realidad observable, } \\
\text { obtenidos mediante el método de investigación científico. }\end{array}$ \\
\hline Bunge & $\begin{array}{l}\text { Indica que conviene delimitar el concepto de tecnología, que para él consiste en el desarrollo de la actividad científica } \\
\text { aplicada al mejoramiento de nuestro medio natural y artificial, a la invención y manufactura de bienes materiales y } \\
\text { culturales. }\end{array}$ \\
\hline Kohan & $\begin{array}{l}\text { El objetivo principal de una ciencia, más que una mera descripción de fenómenos empíricos, es establecer, mediante } \\
\text { leyes y teorías, los principios generales con que se pueden explicar y pronosticar los fenómenos empíricos. }\end{array}$ \\
\hline
\end{tabular}

Fuente: información recabada de Asensi-Artiga y Parra-Pujante (4-5). 
En el seguimiento sistemático de la obra de Asensi-Artiga y Parra-Pujante (6) y Valdés (36), se permite confirmar que hay un proceso histórico y evolutivo de la filosofía de la ciencia que para el caso se intenta rastrear desde el siglo $\mathrm{xv}$, llegando a convertirse en una disciplina autónoma en el siglo XVIII, avanzando su consolidación en el siglo xIx y en el siglo $\mathrm{xx}$, que ha llegado a un proceso de maduración con el Circulo de Viena e intelectuales como Popper y Kuhn, que la impulsan hacia una nueva filosofía de la ciencia

\section{La discusión en torno al método científico}

A lo largo de la historia, el hombre se ha enfrentado a un sinnúmero de obstáculos y problemas para desentrañar los secretos de la naturaleza, tanto para vivir con ella, como de ella, en armonía. Para superar esos problemas ha empleado muy diversas estrategias, las cuales dieron paso a la formalización de procedimientos que, en última instancia, no son sino el propio método científico. Por ello en la línea de Asensi-Artiga y Parra-Pujante (8), Bojacá (186), Suárez (16) y Ortiz y García (110) se diserta en connotación a la definición y consecuencia la relación con filosofía de la ciencia. En el proceso de construcción de la ciencia, de manera elemental han operado dos métodos antagónicos pero complementarios: el método inductivo de lo particular a lo general y el método deductivo de lo general a lo particular.

Por tanto, Bojacá (175) plantea una definición etimológica, de método: (meta odos = a lo largo del camino) se dice del conjunto armónico de medios para lograr un fin, en este caso para logar la ciencia. Existen métodos para aprender ciencia (didáctica), métodos para trasmitir ciencia (pedagogía); métodos para crear ciencia (método científico). Por consiguiente, Asensi-Artiga y Parra-Pujante (8) coinciden en que el método científico rige toda la actividad científica, desde la gestación del problema hasta la difusión del resultado.

Asimismo, Asensi-Artiga y Parra-Pujante (5) definen: "el método científico tiende a reunir una serie de características que permiten la obtención de nuevo conocimiento científico. Es el único procedimiento que no pretende obtener resultados definitivos y que se extiende a todos los campos del saber. El método científico es imprescindible incluso para la superación de los mínimos exigidos para que un trabajo de investigación sea aceptado por la comunidad científica". También afirman, citando a Rudio, que:
El método es un proceso de elaboración consciente y organizado de los diferentes procedimientos que nos orientan para realizar una operación discursiva de nuestra mente. Por ello, las etapas del método científico se corresponden de manera general con las del proceso del pensamiento reflexivo, como son: 1) advertencia, definición y comprensión de una dificultad, 2) búsqueda de una solución provisional, 3) comprobación experimentalmente de la solución adoptada, 4) verificación de los resultados obtenidos, y 5) diseño de un esquema mental en cuanto a situaciones futuras para las que la situación actual será pertinente.

Por lo tanto, Bojacá afirma que:

En el orden empírico, la investigación de define como el proceso sistemático y controlado de obtención, análisis e interpretación de datos que representan determinados hechos o fenómenos a fin de describir (análisis), establecer (planteamiento hipotético) o descubrir los principios y leyes que rigen tales hechos o fenómenos al actuar o comportarse (axiomatización)". (176)

\section{Fases o etapas del método científico}

Asensi-Artiga y Parra-Pujante (5) exponen que: "las etapas mencionadas se corresponden con: 1) formulación del problema que motiva el comienzo de la investigación, 2) enunciado de la hipótesis, 3) recogida de datos, y 4) análisis e interpretación de los datos". Ortiz y García (58-62) difieren en el número de etapas indicando que son: 1) observación, 2) hipótesis y 3) comprobación, pero de cierta forma guardan coherencia y correlación. Un resumen de estos conceptos en la tabla 7:

En la perspectiva de Ortiz y García (58), el método científico es el procedimiento planteado que se sigue en la investigación para descubrir las formas de existencia de los procesos objetivos, para desentrañar sus conexiones internas y externas, para generalizar y profundizar los conocimientos así adquiridos, para llegar a demostrarlos con rigor racional. El método científico también se le caracteriza como un rasgo de la ciencia, tanto de la pura como de la aplicada, y por su falibilidad puede perfeccionarse mediante la estimación de los resultados a los que lleva mediante el análisis directo. El método científico es la lógica general empleada, tácita o explícitamente para valorar los méritos de una investigación. 
Tabla 7. Etapas del método científico

\begin{tabular}{|l|l|}
\hline \multicolumn{1}{|c|}{ ETAPA } & \multicolumn{1}{c|}{ DESCRIPCIÓN } \\
\hline OBSERVACIÓN & $\begin{array}{l}\text { Es una etapa del proceso de investigación científica que reviste gran importancia porque mediante } \\
\text { su proceso, aparte de haber un mayor acercamiento con el objeto de estudio, se está en el momento } \\
\text { de tomar de él, datos que son la base esencial para cuantificar y cualificar a ese objeto de estudio de } \\
\text { manera científica. }\end{array}$ \\
\hline HIPÓTESIS & $\begin{array}{l}\text { La palabra hipótesis deriva de hipo= bajo, thesis=posición situación, en el ensayo de explicación } \\
\text { de ciertos hechos, la supuesta relación que existe entre ellos (lo que está en la base, debajo de una } \\
\text { situación dada que trata de explicarse). Las hipótesis son la medula no solo de la práctica de la } \\
\text { investigación científica, para la que constituyen el instrumento intelectual: principal }\end{array}$ \\
\hline COMPROBACIÓN & $\begin{array}{l}\text { La comprobación científica de hipótesis, ya sea de origen factual proveniente de las ciencias que } \\
\text { estudian hechos, como la física, la química, la biología, o de origen formal como es el cado e la } \\
\text { lógica y de las matemáticas, representan uno de los pasos fundamentales del método científico; en } \\
\text { efecto, la comprobación científica es el paso final de todo proceso de investigación que incluye el } \\
\text { planteamiento del problema, la formulación de una hipótesis, las consecuencias de estas, las técni- } \\
\text { cas de contrastación y la comprobación; esta última es la que determinará la verdad o la falsedad. }\end{array}$ \\
\hline
\end{tabular}

Fuente: información recabada de Ortiz y García (58-62).

Para Karl Popper (13), es claro que la investigación toma como punto de partida los problemas. Para investigar, hay que plantear hipótesis que sirvan como intentos de solución, una vez formuladas hay que comprobar dichas hipótesis y extraer consecuencias para ver si se cumplen o no. Si se cumple, confirman la teoría, y si no se cumplen, la desmientan o falsan. Así, para que una teoría sea aceptada como verdad científica, tiene que poder refutar, es decir, permitir su falsación a través de los hechos (Suárez 12).

El falsacionismo, refutacionismo o principio de falsabilidad es una corriente epistemológica fundada por el filósofo austriaco Karl Popper (1902-1994). Para Popper, contrastar una teoría significa intentar refutarla mediante un contraejemplo. Si no es posible refutarla, dicha teoría queda corroborada, pudiendo ser aceptada provisionalmente, pero nunca verificada. Frente al neopositivismo, Popper calificó su postura de racionalismo crítico. Al respecto, Asensi-Artiga y Parra-Pujante (6) proponen que:

Siguiendo a Einstein, Popper advertía que la naturaleza sólo contesta que no o que quizá a las preguntas del científico, nunca que sí. El progreso científico se da entonces, no a través de la imposible verificación, como pretendían los miembros del Círculo de Viena, sino por la falsación. De la misma manera, la ciencia no procede por inducción, saltando de la observación de casos singulares a la formulación de una ley general. El genuino método científico es hipotético-deductivo.

A partir de la obra del norteamericano Thomas Kuhn (1922-1996), Estructura de las revoluciones científicas (1962), se dio un cambio en la perspectiva y se empezaron a tener en cuenta los aspectos históricos, sociológicos y culturales de la ciencia. Según Kuhn, la estructura de las revoluciones científicas se puede clasificar de descriptiva. Apenas dedica espacio a conceptos como verdad o conocimiento, y presenta la ciencia bajo un enfoque histórico y sociológico.

Para Thomas Kuhn, la comunidad científica es la que permite el avance de la ciencia a través de teorías, llamadas paradigmas o conquistas científicas universalmente aceptadas que durante un tiempo determinado brindan un modelo de problemas y soluciones aceptables para aquellos que trabajan en un campo de investigación (Suárez, 2003).

En perspectiva de Asensi-Artiga y Parra-Pujante (2002), las teorías dominantes bajo las que trabajan los científicos conforman lo que Kuhn llama paradigma. La ciencia normal es el estado habitual de la ciencia, el científico no busca criticar el paradigma, sino que da este por asumido y busca la ampliación del mismo. Y señala que:

Kuhn utilizó la palabra paradigma por primera vez en 1959, en una conferencia sobre 'la tensión esencial', es decir, sobre los aspectos convergentes y divergentes de la empresa científica, que garantizan, respectivamente, la coherencia de lo que luego llamaría sus períodos de ciencia normal y los cambios conceptuales radicales que más tarde caracterizaría como revoluciones científicas.

Si el número o la importancia de problemas no resueltos dentro de un paradigma es muy grande, puede sobrevenir una crisis y cuestionarse la validez 
del paradigma. Entonces la ciencia pasa al estado de ciencia extraordinaria o ciencia revolucionaria en el que los científicos ensayan teorías nuevas. Si se acepta un nuevo paradigma que sustituya al antiguo se ha producido una Revolución Científica.

Contra el método, Paul Feyerabend (1924-1994) llama la atención acerca de la imposibilidad de que haya un método capaz de garantizar el desarrollo del pensamiento científico. Por el contrario, cada vez que avanza una ciencia se violenta el método utilizado y se descuida toso tipo de reglas metodológicas (Suárez, 2003). Y Asensi-Artiga y Parra-Pujante (2002) indican que, por diversas razones, la historia de la ciencia se convirtió para Feyerabend en un ingrediente esencial de la filosofía de la ciencia. Feyerabend advirtió que las reglas de referencia de la ciencia son violadas por los propios científicos, en oposición a las tesis empiristas oficiales. Frente a Popper creía que no hay modo de delimitar, o demarcar - en terminología popperiana- la ciencia de lo que no lo es.

\section{Conclusiones}

Es una cuestión importante, no solo estudiar la diversificación científica sino que también se analice el origen mismo de la ciencia, ya que es una respuesta a la inquietud gnoseológica propia del interés humano que estudia la naturaleza, el origen y el alcance del conocimiento. Una premisa válida en la actualidad, más aun cuando se asiste a la globalización del conocimiento y cuando por causa de la tecnología se infiere una pseudo-adquisición del saber, razón por la cual adquiere un carácter de imperativo el discernimiento sobre elementos de bondad o maldad, importancia o utilidad, entre otros antagonismos que refuerzan el proceso de enseñanza aprendizaje.

La filosofía de la ciencia que se ocupa de investigar el conocimiento científico y la práctica científica, al igual que de hacer seguimiento al desarrollo y evolución de las teorías científicas y se apoya en diversos de sus tratados como la epistemología, la cosmología, la lógica, la ontología y la teoría del conocimiento, entre otros, a fin de consolidar las bases filosóficas la validez y veracidad del conocimiento científico y de las teorías científicas.

Se considera que la ciencia requiere de la filosofía y de sus tratados: gnoseología, epistemología, crítica del conocimiento, para una sólida descripción e interpretación de los fenómenos naturales. Si el siglo xx fue el de la ciencia, sin lugar a dudas, al actual le quedan retos para la ciencia y también para la filosofía.
En la disertación se puede analizar la importancia de la consolidación histórica de la filosofía de la ciencia como autónoma y el desarrollo consecuente de sus postulados. Como punto de partida en el siglo $\mathrm{xv}$ se puede evidenciar el recorrido histórico de la filosofía de la ciencia, con especial acento en el siglo XVII, XVIII y XIX, demostrando su solidez y madurez en el trascurso del siglo xx, con fortaleza en el momento del Círculo de Viena.

El método científico, como camino de investigación científica y la confrontación de la ciencia, en apoyo de sus dos principales métodos: deductivo e inductivo y las diversas etapas o fases permiten consolidar la ciencia y la filosofía. Entre tanto que Karl Popper confronta la falsación versus la verificación de la ciencia necesaria para un proceso científico. Thomas Kuhn, que propone las teorías llamadas paradigmas y las estructuras de las revoluciones científicas y desde luego Paul Feyerabend que se opone a un método eficaz para el desarrollo científico.

\section{Referencias}

Asensi-Artiga, Viviana y Antonio Parra-Pujante. "El método científico y la nueva filosofía de la ciencia”. Anales de Documentación 5 (2002): 9-19. Digital.

Azcárraga, José. “Ciencia y Filosofía”. Métode (2003): 4046. Digital

Bojacá, Jorge. ZYX La lengua filosófica universal. Colombia: Edición A\&C. Impreso.

González, Ángel. Historia de la Filosofía: en Cuadros Esquemáticos. Madrid: Ediciones EPESA, 1969. Impreso.

Ortiz, Frida y María García. Metodología de la investigación: el proceso y sus técnicas. México: Limusa, 2010. Impreso.

Padrón, José. “Tendencias epistemológicas de la investigación científica en el siglo xxi”. Revista Cinta de Moebio 28 (2007): 1-28. Digital.

Real Academia Española. Diccionario Práctico del Estudiante. Barcelona: Talleres Gráficos de Printer, 2007. Impreso.

Suárez, María. Pienso... Filosofía y su historia II. Bogotá: Editorial Voluntad, (2003). Impreso.

Valdés, O. (2015). Filosofía de la ciencia. Memorias de clase. (CD) Digital.

Verano, Leonardo. Pienso... Filosofía y su Historia I. Bogotá: Editorial Voluntad: Educación Media, 2004. Impreso. 